\title{
A Humanização Enquanto Componente da Formação em Saúde: A Visão de Discentes de Graduação
}

Humanization as a Component of Health Education: The Vision of Undergraduate Students

La Humanización Mientras que el Componente de Capacitación en Salud: La Visión de Los Estudiantes de la Graduación

Maria Gicarlia Braz ${ }^{1}$

Margarete Pereira Cavalcante ${ }^{2}$

\section{Resumo}

Objetivo: identificar, sob a ótica de discentes de graduação da área da saúde, qual a contribuição para a sua formação no ensino na saúde, vivenciada nas práticas do projeto de assistência humanizada à mulher e à criança, no período de 2010 a 2013. Método: Trata-se de um estudo exploratório, descritivo, com uma abordagem qualitativa, na qual se utilizou a técnica de entrevista não estruturada para a apreensão dos dados. Teve como sujeitos da pesquisa, 14 discentes de diferentes cursos de graduação da Universidade Federal de Alagoas e do Centro de Estudo Superior de Maceió. A interpretação das falas das entrevistadas foi orientada pela Teoria Análise de Conteúdo de Bardin, como ferramenta para análise dos dados qualitativos. Resultados: o projeto em pauta contribui para a formação de futuros profissionais reflexivos e críticos com o perfil adequado para

${ }^{1}$ Graduada em Enfermagem pela Universidade Regional do Nordeste. Graduada em Fisioterapia pela Universidade Estadual da Paraíba. Especialista em Educação Especial pela Universidade Federal de Alagoas. Autor correspondente: End Campus A.C. Simões. Av. Lourival Melo Mota, s/n. Tabuleiro dos Martins.CEP:57072-900.Maceió - AL. E-mail: gicarlia@hotmail.com

${ }^{2}$ Graduada em Serviço Social pela Universidade Federal de Alagoas. Mestre em Serviço Social pela Universidade Federal da Paraíba.

Recebido: Nov/2016 - Aceito: Mar/2017. 
atuar no Sistema Único de Saúde. Conclusão: A inserção dos discentes em ações de extensão humanizadas, pode resultar na formação de sujeitos comprometidos na transformação das práticas de saúde, agregando novos valores que possibilitam a construção de novos modos de cuidar em sua futura prática profissional.

\section{Descritores: Atenção Primária à} Saúde; Humanização da Assistência; Educação em Saúde.

\section{Abstract}

Objective: to identify from the undergraduate of health area students' point of view, the contribution to their training, experienced in the practices of the project of humanized care to women and children, realized from 2010 to 2013. Methodology: This was an exploratory, descriptive study with a qualitative approach, in which the unstructured interview technique was used to collect the data. As subjects of the research, there were 14 students from different undergraduate courses of the Federal University of Alagoas and the Center of Higher Education of Maceió. The interpretation of the interviewees' speeches was guided by the Content Analysis Theory of Bardin, as a tool for analyzing the qualitative data. Results: indicate that the project in question contributes to the formation of future reflective and critical professionals with the appropriate profile to act in the Unified Health System. Conclusion: the insertion of the students in humanized extension actions, can result in the formation of Subjects involved in the transformation of health practices, adding new values that allow the construction of new ways of caring in their future professional practice.

Descriptors: Primary Health Care; Humanization of Care; Health Education.

\section{Resumen}

Objetivos: identificar desde la perspectiva de los estudiantes de la graduación de la salud, la contribución a su formación en la enseñanza de la salud, con experiencia en la práctica del diseño humanizado para las mujeres y los niños, en el período 2010-2013. Método: Se trata de un estudio exploratorio, descriptivo, con enfoque cualitativo, que se utilizó la técnica de entrevista no estructurada para la toma de datos. Tenía como sujetos de investigación, 14 estudiantes de 
diferentes cursos en la Universidad Federal de Alagoas y Centro de Estudios Superiores de Maceió. La interpretación de las palabras de los entrevistados estuvo guiado por la Teoría Bardin Análisis de Contenido como una herramienta para el análisis de datos cualitativos. Resultados: indican que el proyecto en cuestión contribuye a la formación de los futuros profesionales reflexivos y críticos con el perfil adecuado para trabajar en el Sistema Nacional de Salud. Conclusion: la inclusión de los estudiantes en las acciones de extensión humanizados puede dar lugar a la formación de los sujetos involucrados en la transformación de las prácticas de salud, añadiendo nuevos valores que permiten la construcción de nuevas formas de cuidar en su futura práctica profesional.

Descriptores: Atención primaria de salud; Humanización de la Atención; Educación en Salud.

\section{Introdução}

A humanização da atenção à saúde tem se mostrado um tema de grande interesse e objeto de muitos debates na saúde coletiva brasileira. No entanto, em virtude da vulnerabilidade do paciente e ao confronto entre tecnologia e humanização, as discussões têm dado ênfase à importância do atendimento hospitalar, e pouca relevância no diz respeito à humanização nos atendimentos na atenção básica $^{(1)}$.

A sistematização sobre a humanização na saúde torna-se mais evidente em 1999, após o planejamento e implementação de algumas ações e programas, a exemplo do Programa Nacional de Humanização da Atenção Hospitalar (PNHAH), lançado pelo, o Ministério da Saúde em 2001. A forma precisa que apresentavam essas iniciativas resultava na fragmentação das ações levando a um pensamento vinculado a práticas voluntaristas $\mathrm{e}$ $\operatorname{assistencialistas}^{(2)}$.

Percebendo a necessidade de modificar o atual cenário imposto ao atendimento do SUS, em 2003, o Ministério da Saúde propões ampliar a humanização para além de o ambiente hospitalar, estabelecendo nessa proposta, a Política de Humanização (PNH) ou Política Nacional de Humanização da Atenção da Gestão em Saúde no SUS (HumanizaSUS), almejando com sua transversalidade atingir todos os níveis de atenção de saúde na esfera estadual e municipal ${ }^{(3)}$. 
A estrutura da PNH encontra seus fundamentos em três princípios, a saber: transversalidade, refere-se ao grau de comunicação entre os sujeitos e coletivos e que está relacionado com a dimensão da compreensão do processo de trabalho; a indissociabilidade entre atenção e gestão, ou seja, a inseparabilidade entre o modo de cuidar e modos de gerir e apropriar-se do trabalho e a afirmação do protagonismo, corresponsabilização e autonomia dos sujeitos e coletivos, envolvidos como sujeitos que assumam um lugar central nos acontecimentos de saúde ${ }^{(4)}$.

O conceito de humanização é polissêmico, com referência à atenção em saúde, visto que não há uniformidade de conceito e nem de metodologia, alguns percebem seus sentidos ligados ao humanitarismo, outros como relação interpessoal e outros como políticas públicas.

A humanização como política pública deve criar espaços de construção e troca de saberes, possibilitando mudanças nas práticas de saúde, e não ter um caráter fragmentado em cada iniciativa de atividades propostas ou programas ${ }^{(5)}$.

Neste sentido, a humanização do atendimento supõe encontro entre sujeitos que compartilham saber, poder e experiências vivenciadas. Cabe considerar que o trabalho em equipe tem importância fundamental no processo de organização e reorganização da atenção à saúde no Sistema Único de Saúde (SUS). Em relação às práticas junto à mulher e à criança, assume maior significação, ainda mais quando vinculadas à formação em saúde, no âmbito da universidade.

As novas Diretrizes Curriculares Nacionais dos cursos de graduação em saúde concebem como dimensão fundamental da formação, o trabalho em equipe, na medida em que, para atender aos princípios do Sistema Único de Saúde (SUS), as universidades se tornam responsáveis em proporcionar uma formação comprometida com as necessidades da sociedade ${ }^{(6)}$.

Tendo em vista esses desafios ${ }^{(7)}$, concebem a formação em saúde, na perspectiva do denominado quadrilátero, ao considerar como componentes integrantes dessa formação, o ensino, a gestão, as práticas de atenção e o controle social. Ressalta, ainda, o protagonismo dos profissionais e dos usuários dos serviços de saúde, como fundamentais para a construção e organização de uma educação responsável por desencadear processos e práticas voltadas à transformação da realidade, com vistas a operar 
mudanças, além de “[...] mobilizar caminhos (negociar e pactuar processos) convocar protagonismo (pedagogia in acto) e detectar a paisagem interativa e móvel de indivíduos, coletivos e instituições, como cenário de conhecimentos e invenções (cartografia permanente) ${ }^{,(7: 59)}$.

Nessa perspectiva, entendemos que o perfil profissional necessário para os serviços de saúde inclui a capacidade na formação de profissionais críticos, reflexivos, com compromisso ético, sociopolítico, preparado para enfrentar problemas complexos que se apresentam na sociedade e, mais especificamente nos serviços de saúde. Outro aspecto importante na formação e desenvolvimento de indivíduos capazes de exercer a cidadania plena, dizem respeito à indissociabilidade entre $\mathrm{o}$ ensino, pesquisa e extensão, visto que, a articulação entre ensino, serviço e integração teoria e prática implicam em ações pedagógicas que extrapolam os muros da universidade, indicando a necessidade da inserção do aluno em realidades concretas, fazendo com que a formação seja centrada na prática, em consonância com o mundo do ensino e do trabalho.

O projeto desenvolveu-se em nove Unidades de Saúde da Estratégia da Saúde (ESF) de Maceió, no período de 2010 a 2013, envolvendo trabalhadores, usuários e discentes de diferentes cursos de graduação: medicina, enfermagem, serviço social, odontologia, nutrição e psicologia da UFAL e do Centro de Estudos Superiores de Maceió (CESMAC), e objetivou através de ações estratégicas, contribuir para transformar e qualificar as práticas de saúde, com foco na humanização da assistência à saúde da mulher e à criança, e aproximar o discente dos serviços de saúde e da comunidade, na perspectiva de contribuir na formação profissional voltada para $\mathrm{o}$ fortalecimento da atenção básica à saúde.

\section{Método}

A pesquisa utilizou uma metodologia tipo exploratória, descritiva, numa abordagem qualitativa. Utilizou-se a entrevista não estruturada para a coleta dos dados, por se constituir em importante instrumento utilizado no trabalho de campo, visto que a entrevista não estruturada é uma forma de coleta de dados em que o entrevistador pode explorar mais amplamente uma questão.

A pesquisa teve como amostra 14 discentes de diferentes cursos de graduação: duas de enfermagem, três de 
medicina, três de serviço social, três de psicologia, uma de odontologia e duas de nutrição, da Universidade Federal de Alagoas (UFAL) e do Centro de Estudos Superiores de Maceió (CESMAC), que participaram do projeto intitulado: assistência humanizada à mulher e à criança, realizada em nove Unidades Básicas de Saúde da Estratégia de Saúde da Família de Maceió, no período de 2010 a 2013. O recrutamento e a realização da pesquisa ocorreram por meio de endereço eletrônico. Como critério de exclusão considerou-se as discentes que não foram localizadas por meio do endereço eletrônico e/ou telefone. Para efeito de codificação das falas das discentes envolvidas na pesquisa, foram adotados nomes fictícios de flores, para garantia do sigilo.

As questões que serviram como base orientadora para as entrevistas foram: a) $\mathrm{O}$ que você entende por humanização em saúde? b) Qual a importância do trabalho em equipe na saúde? c) Como as ações humanizadas podem ser realizadas na sua prática nos serviços de saúde? d) Qual a contribuição do projeto na sua formação em saúde?

Após os registros, as entrevistas foram transcritas na íntegra, constituindo-se em material sobre o qual se realizou uma leitura cuidadosa para a apropriação dos significados. A interpretação das falas foi orientada pela teoria de análise de conteúdo de Bardin, por considerá-la como a técnica de análise de material qualitativo capaz de descrever o conteúdo explícito ou implícito no processo de comunicação, seja ele por meio de falas ou de textos, dos sujeitos envolvidos na investigação.

\section{Resultados e Discussão}

Nos resultados da pesquisa, percebeu-se que as discentes atribuíram diferentes sentidos para a humanização em saúde - alguns que se distanciam e outros que aproximam da humanização, como as políticas públicas de saúde. Elas concebem a humanização em saúde, ora voltada para o cunho de respeito humano, bem-estar social, ora a entendem como um método ou um modo diferente de prestar assistência.

\footnotetext{
Humanização em saúde é um modo diferenciado no tratamento de pacientes, ou seja, é um método que trata a pessoa com a doença e não só a doença da pessoa (Protea).

É transpor os sentimentos através das palavras trazendo o atendimento e relacionamento com o próximo para o campo da comunicação dando a palavra não só ao usuário como ao profissional a fim de tornar a saúde um todo (Jasmim).
}

Tal como o próprio nome diz, 
abrange tornar humana a assistência prestada tanto clinicamente, no exercício da profissão, quanto emocionalmente, [...] trabalha a emoção e a forma como abordar determinado tema (Violeta).

Os relatos acima expressam uma perspectiva teórica de humanização fundamentada nos princípios que a consubstancia, ao afirmar que para humanizar a relação entre serviço, profissional de saúde e usuário, não basta considerar a questão da responsabilidade e do respeito como pressupostos para a realização da assistência $^{(1)}$. Segundo os autores referidos, na perspectiva da $\mathrm{PNH}$, na humanização das práticas de atenção e gestão deve-se levar em conta a humanidade como força coletiva que impulsiona e direciona o movimento das políticas públicas.

Ainda em conformidade com o tema, as participantes da pesquisa apresentaram uma reflexão relevante sobre a humanização em saúde, que vai ao encontro da Política Nacional de Humanização, como segue abaixo.

A humanização dos serviços de saúde vem com a proposta de redesenhar as práticas de cuidado, refletindo sobre as relações de trabalho, sistema de gestão e os serviços prestados a sociedade (Gardênia).

Humanização da saúde é um acesso integral, igualitário no que diz respeito ao acesso, porém individualizado a cada ser, multiprofissional (Palma).

[...] é o atendimento, respaldado pelo comportamento ético, aliado com o conhecimento técnico e cuidados voltados para as necessidades dos usuários (Rosa).

Os relatos acima expostos encontram-se em consonância ${ }^{(5)}$, quando asseguram que humanizar a atenção e a gestão em saúde no SUS se apresenta como meio para a qualificação das práticas de saúde: acesso com acolhimento; atenção integral e equânime com responsabilização e veículo; valorização dos trabalhadores e usuários, com avanço na democratização da gestão e no controle social participativo.

Também foi possível identificar e sistematizar os diferentes significados e entendimentos no que diz respeito à humanização da assistência, trazidos pelos depoimentos. $\mathrm{Na}$ compreensão de algumas discentes, a humanização em saúde foi enfocada como um método de tratar pessoas; é transpor sentimentos, trabalhar emoções. Outras enfocam aspectos relacionados ao acesso integral, ao atendimento respaldado pelo comportamento ético, acessibilidade aos serviços, e também como uma proposta de redesenhar as práticas de cuidado. 
Neste sentido, torna-se necessário estabelecer relação terapêutica entre usuário e os profissionais de saúde, de maneira a humanizar o atendimento. Porém essa relação não deve ser entendida como um ato de caridade exercido por profissionais abnegados e já dotados de qualidades humanas essenciais. A humanização deve ser compreendida como resultado de novas práticas no modo de fazer trabalho em saúde, levando em consideração o trabalho em equipe, adicionando-se a troca de saberes entre usuários e profissionais, alicerçados em ações guiadas pela compreensão e pela valorização dos sujeitos como reflexo de uma atitude ética e humana, a ser entendida como direito humano à saúde ${ }^{(8-9)}$.

As discentes fizeram referência à importância do trabalho em equipe; destacaram que o compartilhamento de saberes é fundamental para garantir a organização dos serviços e melhorar a qualidade da assistência em saúde, conforme disposto abaixo:

No trabalho em equipe multiprofissional há a necessidade de uma inter-relação entre os diferentes profissionais que devem ver o paciente como um todo, de forma humanizada (Girassol).

O trabalho em equipe garante maior organização nos serviços $e$ permite vários olhares e várias formas de atuação sobre as diversas situações, integrando todos os envolvidos em ações em prol de toda a comunidade (Margarida).

Diminuição da distância no relacionamento dos profissionais com a comunidade, a divisão das responsabilidades do cuidado entre a equipe, a cooperação $e \quad a$ colaboração dos envolvidos na execução dos trabalhos para que os objetivos comuns sejam alcançados (Rosa).

O trabalho em equipe representa um processo de relação a ser pensado entre os sujeitos envolvidos, de forma que no cotidiano do trabalho a horizontalidade das relações possa estabelecer-se, viabilizando a melhoria dos serviços nos modelos de atenção à saúde ${ }^{(10)}$. Assim, o trabalho em equipe é indispensável, pois poderá melhorar a qualidade da educação, permitindo uma maior e melhor adequabilidade ao singular e às características peculiares dos diferentes grupos de alunos ${ }^{(11: 158)}$.

Considera-se que as discentes reconheceram a importância da vivência durante o desenvolvimento das ações de educação em saúde, o que possibilitou ampliar seus conhecimentos com competência para trabalhar em equipe multiprofissional, compartilhando saberes e experiências e, também, a formação de vínculo com a comunidade na atenção básica de saúde.

As discentes concebem que as ações humanizadas podem estar 
presentes na sua prática nos serviços de saúde, desde a intervenção individual, como nas ações coletivas. Fazem referência à importância do trabalho em equipe para o fortalecimento de ações que possam promover a integralidade da assistência

As ações de humanização podem estar presentes em toda prática profissional, desde a intervenção individual, com o usuário até ações coletivas, e também nas discussões da equipe multiprofissional e da gestão para fortalecer essas ações (Gardênia).

O profissional de saúde deve deixar de lado as práticas que tratam do individuo apenas como um ser biológico, [...] deve estar atento ao cuidado integral à saúde do indivíduo, levando em consideração seu estado psicológico e social (Hortência).

Os serviços de saúde somente têm condições de fluir se houver trabalho em equipe. Não se faz saúde com apenas uma categoria profissional (Tulipa).

A humanização como política pública deve criar espaços de construção e troca de saberes, que possibilitem mudanças nas práticas de saúde, e não deve apresentar caráter fragmentado em cada iniciativa de atividades propostas ou programas ${ }^{(5)}$. Ainda em consonância com os relatos $\operatorname{apresentados}^{(12: 222)}$, afirmam que “[...] não só os aspectos biológicos, mas também psíquicos, sociais, ambientais, éticos estão implicados necessariamente nos processos de saúde e doença, influenciando, portanto, diretamente nas possibilidades de tratamento e cura". Desse modo, a humanização pode ser compreendida como resultado de novas práticas de saúde que, quando desenvolvidas na perspectiva da interdisciplinaridade, poderão assegurar a integralidade da assistência no campo da atenção à saúde.

Com base na experiência desenvolvida, as discentes ressaltam que o aprendizado e as oportunidades vivenciadas durante a realização do projeto foram importantes para a sua formação em saúde e crescimento profissional, uma vez que proporcionam a aproximação com a comunidade e o compartilhamento de saberes e experiências entre diversas áreas de saberes por meio de ações preventivopromocionais em saúde, com aprendizagem de habilidades e competência para trabalhar em equipe multiprofissional, com caráter generalista, humanista e crítico da realidade da saúde atual.

A extensão vem com o papel de contribuir na lacuna na formação, ampliando o conhecimento do estudante e proporcionando viver a prática, como funciona os serviços e como são realizadas as ações. $O$ projeto [...] propiciou a formação de vínculos com a comunidade e os profissionais dos serviços (Gardênia). 
Contribuiu na minha formação enquanto profissional generalista, humanista e critico, me permitindo um novo olhar sobre a atenção primária à saúde da população, no desenvolvimento de minha capacidade de trabalhar em equipe multiprofissional, de forma crítica e reflexiva e com competencia social, técnico-científica (Girassol).

Nessa direção, considera-se que as Universidades ainda não estão priorizando a formação de profissionais sensíveis com as condições de vida da população. Afirmam ainda que, na área da saúde, nas instituições formadoras públicas e privadas, a organização curricular tem por base um conjunto de disciplinas isoladas e estanques, reproduzidas de forma acrítica e ahistórica, e que a integração entre ensino e serviço dá-se de forma desarticulada $^{(12)}$.

Neste sentido, o projeto de extensão desempenhou um importante papel no processo educativo e cultural das discentes, na medida em que possibilitou a aproximação do ensino, serviço e comunidade. As Unidades Básicas de Saúde contribuíram como espaço de construção e compartilhamento de saberes, envolvendo graduandos e profissionais das diversas áreas do conhecimento e comunidade. Colaborou ainda, para a formação de futuros profissionais, como sujeito ativo, crítico, reflexivo, criativo e solidário, com competência para trabalhar em equipe, priorizando a integralidade da atenção, incluindo a capacidade de atuar em ambientes complexos, com competência para lidar com as mudanças enfrentadas pelo setor saúde.

\section{Conclusão}

Os resultados da pesquisa identificaram uma possível polissemia de termos atribuídos à humanização em saúde. Na compreensão de algumas discentes, a humanização em saúde foi percebida com um método de tratar pessoas, de transpor sentimentos, de trabalhar emoções; outras enfocaram aspectos relacionados ao acesso integral, ao atendimento respaldado pelo comportamento ético, à acessibilidade aos serviços, e também como proposta de redesenhar as práticas de cuidado.

Nos termos conferidos nas diferentes interpretações apresentadas pelas discentes no campo da produção de saúde, há alguns sentidos que afastam e outros que vão ao encontro do conceito da humanização como políticas públicas. A experiência propiciada pelo projeto de extensão possibilitou um novo redirecionamento no olhar das discentes, de forma 
reflexiva, visualizando a humanização da assistência como direito à cidadania.

Os resultados evidenciados nas falas das discentes encontram fundamentos nas propostas apresentadas pelo projeto de extensão intitulado: "Assistência humanizada à mulher e à criança", que expressa à valorização dos campos de práticas como espaços potenciais de formação para atuar de forma a garantir a integralidade da atenção à saúde.

\section{Referências}

1. Simões ALA, Rodrigues FR, Tavares DMS, Rodrigues LR. Humanização na saúde: enfoque na atenção primária. Enfermagem (Florianópolis) [periódico na internet]. 2007 [citado 2016 abr. 15]; 16(3): 43944. Disponível em: http://www.scielo .br/pdf/tce/v16n3/a09v16n3.pdf.

\section{Morschel A, Barros MEB.} Processos de trabalho na saúde pública: humanização e efetivação do Sistema Único de Saúde. (São Paulo) [periódico na internet]. 2014 [citado 2016 fev. 13]; 23(3): 928-41. Disponível em: http://www.scielo.br/ scielo.php?.

3. Fortes PAC. Ética, direitos dos usuários e políticas de humanização da atenção à saúde. (São Paulo) [periódico na internet]. 2004 [citado 2016 abr. 15]; 13(3): 30-5. Disponível em:http://www.revistas.usp.br/sausoc/a rticle/viewFile/7123/8597.
4. Nora CRD, Junges JR. Política de humanização na atenção básica: revisão sistemática. (São Paulo) [periódico na internet]. 2013 [citado 2016 fev. 16]; 47(6): 86-200. Disponível em: http://www.scielo.br/ pdf/rsp/v47n6/0034-8910-rsp-47-0601186.pdf.

5. Benevides R, Passos E. A Humanização como dimensão pública das políticas de saúde. (Rio de Janeiro) [periódico na internet]. 2005 [citado 2016 fev. 13]; 10(3): 561-71. Disponível em: http://www.scielo.br /pdf/asc/v10n3/a14v10n3.

6. Bispo EP, Tavares CHF, Tomaz JMT. Interdisciplinaridade no ensino em saúde: o olhar do preceptor na Saúde em Família. (Botucatu) [periódico na internet]. 2014 [citado 2016 set. 21]. Disponível em: http://pesquisa.bvsalud.org/saudepubli ca/resource/pt/eps-5233.

7. Ceccim RB, Feuerwerker LCM. O Quadrilátero da formação para a área da saúde: ensino, gestão, atenção e controle social. (Rio de Janeiro) [periódico da internet]. 2004 [citado 2016 fev. 14]; 14(1): 41-65. Disponível em: http://www.scielo.br/pdf/physis/v $14 \mathrm{n} 1 / \mathrm{v} 14 \mathrm{n} 1 \mathrm{a} 04$.

8. Benevides R, Passos E. Humanização na saúde: um novo modismo? (Botucatu) [periódico da internet]. 2005 [citado 2016 jul. 31]; 9(17): 389-406. Disponível em: http://www.scielo.br/pdf/asc/v1on3/a1 $4 \mathrm{v} 1 \mathrm{n} 3$.

9. Casate JC, Corrêia AK. A humanização do atendimento em saúde: conhecimento veiculado na literatura de enfermagem. Enfermagem (Ribeirão Preto) [peiródico da internet]. 2005 [citado 2016 fev. 13]; 13(1): 105-11. Disponível em: http://www.scielo.br.www.scielo.br/pd f/rlae/. 
10. Leite RFB, Veloso TMG. Trabalho em Equipe: representações sociais de profissionais do PSF. Psicologia (Brasília) [periódico da internet]. 2006 [citado 2016 abr. 17]; 28(2): 374-89. Disponível em: http://www.scielo.br//scielo.php?script $=$ sci_abstract\&pid=1414.

11. Souza FP, Monteiro KJ, Elgues GBZ. A avaliação no nível de habilidades para trabalhar em equipe entre professores universitários. Psicologia (São Paulo) [periódico da internet]. 2007 [citado 2016 fev. 23]; 12(2): 154-7. Disponível em: http://www.scielo.br/pdf/pusf/v12n2/v 12n2a04.pdf.

12. Casate JC, Corrêia AK. A humanização do cuidado na formação dos profissionais de saúde nos cursos de graduação. Enfermagem (São Paulo) [periódico da internet]. 2012 [citado 2016 mar. 20]; 46(1): 219-26. Disponível em: http:/www.scielo.br/ pdf/reeusp/v46n1/v46n1a29.pdf. 